\title{
Streptococcus viridans Group
}

National Cancer Institute

\section{Source}

National Cancer Institute. Streptococcus viridans Group. NCI Thesaurus. Code C86811.

A non taxonomic grouping of species within the Streptococcus genus that are alpha hemolytic and do not express Lancefield antigens. 